\title{
FOU YUSUF V IBN AHMAD, REI DE GRANADA, L'INFANT COIX DE LES CRÒNIQUES CASTELLANES?'
}

\author{
ROSER SALICRÚ I LLUCH \\ Institucio Mild $i$ Fontanals \\ (CSIC, Barcelona)
}

\begin{abstract}
SUMARI
1. Estat actual de les investigacions.- 2. L'aportació d'Ibn Asim.- 3. Noves dades arxivistiques de Yusuf V.- 4. La inconsistència del suposat Muhammad X ibn Utman el Coix.- 5. Fou Yusuf ibn Ahmad l'infant Coix de les cròniques? Nihil obstat.- 6. Yusuf $\mathrm{V}$ ibn Ahmad el Coix, rei de Granada: reordenació de les dades i relectura del periode.
\end{abstract}

Fou Yusuf V ibn Ahmad, rei de Granada, l'infant Coix de les croniques castellanes?

Aquesta pregunta sembla, d'entrada, del tot fora de lloc. Pero la conjunció d'una sèrie de dades disperses del trenca-closques del panorama polític granadí de mitjan segle XV que, seguint la interpretació fins ara considerada com a valida, encaixen de manera forçada, la converteix, si

\footnotetext{
- Aquest article forma part dels estudis realitzats dins del Projecte d'Investigació titulat La Corona d'Aragó, frontera aimb l'Islain. Institucions $i$ societal (segles XIV $i$ XV) (núm. PB910141), aprovat i finançat per la Direcció General d'Investigació Científica i Tźcnica (DGICYT) del Ministeri d'Educació i Ciencia (MEC). La meva recerca al Departament d'Estudis Medievals de la Institució Mila i Fontanals del CSIC ha estat possible gracies a una beca pre-doctoral de Fonnació de Personal Investigador del MEC, i ha comptat també amb l'ajut d'una beca d'investigació del Programa d'Estudis Catalans "Joan Maragall", convocatoria de 1994, patrocinat conjuntament per la Fundació José Ortega i Gasset i la Fundació "la Caixa".

Abroviatures utilitzades: $\mathrm{ACA}=$ Arxiu de la Corona d'Aragó; C. = Cancelleria; MEAH = Miscelánea de Estudios Árabes y Hebraicos; reg. = registre.
} 
més no al meu entendre, en una hipdtesi plausible i fins i tot convincent, a la que caldria donar una resposta afirmativa.

La historiografia ha identificat, tradicionalment, aquest infant Coix de Granada de les croniques castellanes amb un Muhammad a qui, segons l'estat actual de les investigacions, correspondria el dese lloc en la sèrie dels sultans granadins, mentre que, per altra banda, ha assimilat Yusuf ibn Ahmad, a qui correspon el cinque lloc en la sèrie dels sultans granadins, amb l'Aben Ismael de les croniques castellanes i de la documentació d'arxiu cristiana.

L'existència d'un Muhammad el Coix s'ha acceptat sempre, pràcticament sense reserves, tot $\mathbf{i}$ que el curs de les investigacions hagi anat reduint, paulatinament, les suposades evidencies i l'extensio temporal de la seva hipotetica permanencia al poder.

$\mathrm{Si}$, de primer, en part a causa de la seva homonímia, s'havia estès el seu regnat fins al perfode cronologic que ara sabem que correspon al darrer regnat de Muhammad IX, l'Esquerra' ', sense solució de continuïtat des de la data del seu suposat ascens al poder, a l'inici de 1445, i s'havia confos també amb el que ara s'ha identificat com a Muhammad XI, el Chiquito -o rei Xic a les fonts catalano-aragoneses-, enllaçant-lo amb la pujada al poder de Sad, despres, davant l'evidencia documental i per conciliar totes les dades disponibles, se li han atribuït dos regnats ${ }^{2}$.

En canvi, mentre que les evidencies cada cop més difícils de conciliar del regnat de Muhammad el Coix s'han anat reduint, l'existencia d'un Yusuf V ibn Ahmad ibn Yusuf II, irrefutable des de la publicacio, el 1914, d'un trasllat d'un document àrab del 15 d'agost de 1445 expedit per la seva cancelleria ${ }^{3}$, ha anat esdevenint progressivament més concreta amb l'apari-

\footnotetext{
'En aquests moments considerat de finals de 1447 fins a mitjan 1453; cf. Jose-Enirique LOPEZ DE COCA CASTANNIR, Revisión de una década de la historia granadina, "MEAH" XXIX-XXX (1980-1981), pp. 61-90, en especial pp. 68 i 78. vàlides.

${ }^{2}$ Vegeu a J.-E. LOPEZ DE COCA, Revisión, les dades que actualment es consideren com a

${ }^{3}$ Mariano GASPAR REMIRO, Translado sinple de nonbramiento para la alcaydia de Almería puesto por el moro de Granada en 1445, en Con motivo del Romancero, "Revista del Centro de Estudios Históricos de Granada y su Reino" IV (1914), pp. 146-148, reedició de "Revista de Libros" II (1914), pp. 8-11; citarem, en endavant, segons la "Revista del Centro de Estudios Históricos".
} 
ció de documentació cristiana d'arxiu ${ }^{4}$ que, al revés d'allo que succeeix amb Muhammad el Coix, ha anat estenent la seva importància.

Per conciliar les contradiccions existents amb l'aparici6, el 1914, del sultà Yusuf $\mathrm{V}$ (fins aleshores desconegut perque, aparentment, passava inadvertit per les croniques cristianes) dins el període cronologic en que s'emmarcava Muhammad el Coix, Seco de Lucena assimila, no sense reserves i gairebé per eliminacio, el Yusuf de nova aparició amb l'Aben Ismael de les croniques ${ }^{5}$-i actualment també de la documentació d'arxiu cristia$\mathrm{na}^{6}$-, sense qüestionar, en cap moment, l'existencia d'un Muhammad el Coix; posteriorment, considerà, de manera provisional a causa de les incoherències cronologiques i per exclusió d'altres possibilitats, que aquest $\mathrm{Mu}$ hammad X el Coix era fill de Muhammad VIII el Petit ${ }^{7}$, $i$ que a ell corresponia la moneda descoberta el 1908 per Gaspar Remiro; ;inalment, el 1959, després de la publicació per part de Torres Fontes de dos documents de l'Arxiu Municipal de Múrcia que acrediten un Muhammad Chiquito el 1451 i $1452^{9}$ i de la seva identificacio amb el fill de Muhammad VIII ${ }^{10}$, Seco de Lucena, tot $\mathrm{i}$ reconeixent que no existia cap testimoni arab que acredités Muhammad X el Coix com a monarca granadi, establi, atenent-se a allo que, en teoria, expliquen els cronistes castellans, que era fill de l'infant Utman, germà de Muhammad IX l'Esquerrà, i, per tant, nebot seu ${ }^{11}$.

\footnotetext{
${ }^{4}$ Vegeu José Hinojosa Montalvo, Las relaciones entre los reinos de Valencia y Granado durante la primera mitad del siglo XV, dins Estudios de Historia de Valencia, Valencia, 1978, pp. 127 i 140-142, documents 18 i 19; J.-E. LOPEZ DE COCA, Revisión, Pp. 64-65.

${ }^{3}$ Luis SECO DE LUCENA PAREDES, Una rectificación a la historia de los ültimos nasries, "Al-Andalus" XVII (1952), 153-163.

'J.-E. LOPEZ DE COCA, Revisión.

${ }^{7}$ Luis SECO DE LUCENA PAREDES, Nuevas rectificaciones a la historia de los nasries, "AlAndalus" XX (1955), pp. $381-405$.

"Mariano GASPAR REMIRO, Una rectificación a la genealogia de los reyes nazarles de Granada, 1908.

'Juan TORRES FONTES, Fajardo el Bravo, Múrcia, 1944 (fins al moment, no ens ha estat possible consultar aquesta obra).

${ }^{10}$ Tal i com, a posteriori, han refermat les investigacions i les noves dades aportades pel mateix Juan TORRES FONTES, La intromisión granadina en la vida murciana (1448-1452), "Al-Andalus" XXVII (1962), pp. 105-154; per Harold LIVERMORE, El segundo Rey Chico, Muhammad XI, y la sucesión de la casa de Abu Nasr Sad, 1452-1456, "Al-Andalus" XXVIII (1963), pp. 331-348; i per J.-E. LOPEZ DE COCA, Revisión, pp. 70-85.

"Luis SECO DE LUCENA PAREDES, Más rectificaciones a la historia de los últimos nasries. Un sultán llamado Muhammad "el Chiquito", "Al-Andalus" XXIV (1959), pp. 275-295, i en concret, per a l'establiment de la genealogia del Coix, p. 281.
} 
Fins a l'actualitat, s'ha seguit acceptant l'existencia de Muhammad el Coix, que era fill d'Utman i que Yusuf $\mathrm{V}$ era l'Aben Ismael de les croniques i dels documents castellans.

La troballa, en el curs de l'elaboració de la meva tesi doctoral sobre les relacions entre la Corona d'Aragó i el regne de Granada al llarg de la primera meitat del segle XV (regnats de Ferran d'Antequera i d'Alfons el Magnànim), de noves dades documentals, a l'Arxiu de la Corona d'Aragó i a l'Arxiu del Regne de València, que no concordaven o que posaven en qüestio algunes de les que fins ara han estat comunament acceptades $i$ que m'obligava a revisar-les, i la seva conjuncio, amb total acord, amb les dades que recentment s'han donat a coneixer a partir del Yunnat al-Rida d'Ibn Asim de Granada, obra arab de mitjan segle XV que fins fa poc es donava per perduda $\mathrm{i}$ que evidencia que Yusuf $\mathrm{V}$ i Ismail eren dues persones diferents ${ }^{12}$, permet posar en dubte moltes de les hipdtesis fins ara vigents.

\section{Estat aCtuAl DE LES INVESTIGACIONS ${ }^{13}$.}

Des de la publicació de la Revisión de una década de la historia granadina per part de López de Coca, considerant sempre que Yusuf V era l'Aben Ismael de les croniques i de la documentació cristiana i l'existència de Muhammad X el Coix ibn Utman ibn Nasr ibn Muhammad V, es creia que Yusuf Aben Ismael, senyor d'Almeria des de 1440-1441 segons consta a la documentació valenciana, havia protagonitzat, el 1444, una revolta

\footnotetext{
${ }^{12}$ Cal esmentar, en primer lloc, la tesi doctoral de Milouda Charouitn HasnaOUi, Edición y estudio del Kitab Yunnat al-Rida de Ibn Asim de Granada, Madrid, Universitat Complutense, 1988 a qui he d'agrair que hagi consentit que usi i citi l'estudi introductori de la seva tesi; Miguel Angel LADERO QUFSADA, Granada. Historia de un país islámico (1232-1571), Madrid, 1989 (3a. edició revisada i ampliada), pp. 181-182 i 189, ja se'n feia ressò; en paral·lel, la mateixa obra fou publicada per Salah YARRAR, Yunnat al-rida fi l-taslim li-ma qadara Allah wa-qada / Abu Yahya Muhammad ibn Asiin Al-Gamati, Ainman, 1989. Posterioment, s'han donat a coneixer algunes de les modificacions introduĩdes per l'obra de Ibn Asim a Joaquín VALLVE, Cosas que pasaron en el Reino de Granada hacia 1448, "Boletín de la Real Academia de la Historia" CLXXXIX (1992), pp. 250-260, i a Milouda Charouit HasnaOUI, Nuevos datos sobre los últimos nasries extraidos de una fuente d́rabe: Yunnat al-Rida de Ibn Asim, "Al-Qantara" XIV (1993), pp. 469-477. Vegeu també Luis SECO DE LUCENA PAREDES, Los Banu Asim intelectwales y políticos granadinos del siglo XV, "MEAH" II (1953), pp. 514.

${ }^{13}$ Vegeu J.-E. LÓPEZ DE COCA, Revisión, 64-75.
} 
interna contra el seu oncle Muhammad IX, i que Muhammad ibn Utman el Coix havia anat a reprimir-la.

Per aquest motiu, s'havia hagut concloure que, en contra del que es pensava fins aquell moment, Yusuf Aben Ismael residr al regne de Granada fins el 1444 , i que fou aleshores, davant del fracàs del seu pronunciament contra l'Esquerrà, quan es refugià a Castella, a la cort de Joan II.

Poc després, Muhammad el Coix hauria protagonitzat un cop d'estat contra el seu oncle, l'hauria destituït i empresonat.

Ibrahim ibn Abd al-Barr i el 'partit abencerratge', refugiats a Montefrio, haurien reclamat la presencia de l'infant Yusuf $A$ ben Ismael, refugiat a Castella, que, amb el suport castella, hauria estat al front de Granada ja a l'agost de $1445 \mathrm{i}$, almenys, fins a principis de 1447; des d'aleshores fins a l'octubre de 1447, quan consta que l'Esquerrà ja havia recuperat el tron, Muhammad X el Coix hauria pres de nou el poder expulsant a Yusuf Aben Ismael i, poc després, ell mateix hauria estat desplaçat pel seu oncle, l'Esquerrà.

Com a aspirant al tron, al llarg de l'estiu de 1446 Muhammad el Coix hauria conquerit les places de Benzalema i Benamaurel i, al llarg del de 1447, Arenas, Huéscar, Vélez Blanco i Vélez Rubio.

Per una banda, s'ignorava si, un cop recuperades les regnes de Granada per Muhammad IX, Yusuf Aben Ismael, que havia de reapareixer el $1450 \mathrm{amb}$ el recolzament de Castella i encara, més endavant, en epoca de Sad, s'havia tornat a refugiar a Castella i, per una altra, es constatava que, des de mitjan 1447, Muhammad X el Coix havia desaparegut d'escena en circumstàncies desconegudes.

\section{L'APORTACIÓ D'IBN ASIM}

Pero, tal com ja recolli Ladero a la tercera edició de Granada. Historia de un pals islámico ${ }^{14}$, la tesi doctoral de Milouda Charouiti modifica substancialment, seguint l'autor que edita, el relat d'aquests anys.

En primer lloc, segons Ibn Asim, Yusuf $\mathrm{V}$ i Abu I-Walid Ismail eren dues persones diferents.

${ }^{14}$ M.Á. LADERO, Granada, notes de les pp. 181-182 i 189. 
Yusuf V s'hauria sublevat directament a Almeria, obligant Muhammad IX a abdicar i a residir a Salobreña. Aleshores, després d'una primera entrada fracassada d'Abu I-Walid Ismail, infant granadí refugiat a Castella, els abencerratges haurien reclamat la seva presència.

Abu l-Walid Ismail hauria intervingut tres vegades a la política granadina. Primer, el 1445; després, el febrer de 1446, apoderant-se de l'Alhambra mentre Yusuf fugia a Almeria, on hauria governat fins al seu assassinat, l'agost del 1447; i, finalment, després d'haver-se tornat a refugiar a Castella, el 1450, alçant-se a Màlaga.

Segons Ibn Asim, Ismail morf el juny d'aquest darrer any i Yusuf $V$ ho hauria fet l'agost del $1447^{15}$.

Aixo implica que el Yusuf de 1462-1463 no pot ser ni Yusuf V ibn Ahmad ni Abu I-Walid Ismail i, com que la Yunna arriba fins a 1450 i Ibn Asim mori el $1453^{16}$, aquest possible Yusuf VI queda, de moment, pendent d'identificacio ${ }^{17}$.

La Yunna, per tant, coincideix amb les fonts cristianes en el fet que Yusuf $V$ fou proclamat el 1445 , pero demostra que no era Ismail.

Segons les fonts castellanes, Yusuf era fill de l'infant Ahmad ibn Yusuf II, és a dir, nebot col'lateral en segona generació o, de fet, fill d'un cosí germà de Muhammad IX. En canvi, segons Ibn Asim, Yusuf V era nebot directe de l'Esquerrà, perquè era fill de la seva germana Fatima alHurra ${ }^{18}$.

Ibn Asim afirma que Fàtima, la germana de l'Esquerrà i mare de Yusuf V, tingué un paper important per intentar mantenir la calma a Granada abans de la rebel-lio del seu fill.

Segons el literat granadi, Yusuf, que residia a Granada, tingué tractes amb els enemics del seu oncle $i$, aleshores, els problemes en les relacions mútues feren que se n'anés a viure a Huétor Vega. Fàtima intentà calmar-lo i proposà al seu germà que nomenés Yusuf alcaid d'Almeria. L'Esquerrà ho acceptà, i aixo permeté que hi haguessin uns anys de calma. Pero les calúmnies dels adversaris de Muhammad IX no cessaren i Yusuf, final-

\footnotetext{
181 .

${ }^{15}$ M. CharoutT, Edición y estudio, pp. 156-168. M.Á. LADERO, Granada, nota de la p.

${ }^{16}$ M. CharoutT, Edición y estudio, pp. 71, 113 i 168.

${ }^{17}$ M. CharouitT, Edición y estudio, p. 168. M.Á. LADERO, Granada, nota de la p. 189, es pregunta si Yusuf VI podria ser el fill de Sad rehena a Castella des de 1455.

${ }^{18}$ M. CharouitT, Edición y estudio, p. 156.
} 
ment, reclutà forces contra el seu oncle, encunyà moneda i s'apoderà de tots els ports fronterers i viles del regne.

La rebel-lió de Yusuf tingué lloc a Almeria, que l'Esquerrà assetjà, personalment, durant un mes. Després de diversos episodis militars i de múltiples sublevacions, Muhammad IX acabà abdicant voluntàriament. Amb Yusuf a l'Alhambra i després de la rendició dels partidaris del seu oncle, refugiats a Moclín, Muhammad IX fou conduït presoner a Salobreña i, després, a Almeria ${ }^{19}$.

Per un altre costat, segons Ibn Asim Muhammad X fou Muhammad ibn Muhammad VIII ibn Yusuf III ibn Yusuf II, és a dir, un fill de Muhammad VIII el Petit, que aparegué a la vida política granadina el 1447, com a braç dret de Muhammad IX, quan aquest acabava de recuperar el tron. La gran entesa entre tots dos féu que es casés amb Umm al-Fath, filla de l'Esquerrà. La seva intervenció en activitats militars es limità a Jaén i Baena, d'on sort victoriós i amb molt de botl, i després a Antequera i a Huéscar. Sempre segons Ibn Asim, les seves múltiples victories haurien obligat els cristians a sol-licitar una treva ${ }^{20}$.

Finalment, la Yunna descobreix que Benamaurel, Benzalema, Arenas, Huéscar i un dels Vélez -no pas tots dos-, conquestes que, a partir de les fonts cristianes, s'atribuïen a Muhammad X el Coix, foren obra de Yusuf $\mathrm{V}^{21}$.

\section{Noves DADES ARXIVISTIQUes DE Yusuf V.}

Basant-se en els documents publicats per Hinojosa, López de Coca ja havia deduiit que Yusuf ibn Ahmad era senyor d'Almeria l'agost de 1440 i de $1441^{22}$. Perd altres documents valencians i catalans l'acrediten ja com a tal (encara que no se'n doni el nom) almenys des de la fi de 1438 .

El 12 de novembre de 1438, gràcies a una carta de Joan Mercader, batlle general del regne de Valencia, tenim not`cia d'un convers de llinatge

${ }^{10}$ M. ChaROUI'T, Edición y estudio, pp. 156-161.

${ }^{20} \mathrm{M}$. Charouit7, Edición y estudio, pp. 169-170. Es tracta de la treva de 1452; vegeu J.E. LOPEZ DE COCA, Revisión, pp. 76-77, i la bibliografia que s'hi cita a les notes.

${ }^{21} \mathrm{M}$. ChAROUITT, Edición y estudio, pp. 183-186.

22J.-E. LOPEZ DE COCA, Revisión, pp. 64-65; cf. J. HINOJOSA, Las relaciones, pp. 127 i 140-142, documents 18 i 19. 
de negres, anomenat Cilim mentre era musulmà, que havia estat amb anterioritat al servei de l'infant d'Almeria ${ }^{23}$, i també sabem que, el 1440, l'infant d'Almeria havia enviat un missatger a la reina Maria, lloctinent general d'Alfons el Magnànim².

Tot aixo encaixa perfectament amb els esdeveniments narrats per Ibn Asim i els aporta una relativa concreció cronologica, concreció que, aparentment, segons es pot deduir de l'estudi introductori de la tesi de la Dra. Charouiti, és absent de la.Yunna pel que fa al perfode almerienc de Yusuf anterior a 1445.

A més, a partir d'una carta que, el desembre de 1443, la reina Maria adreçà a un Mahomet ben Iuceff, infant de Granada a Almeria ${ }^{25}$, podem suposar que Yusuf tenia un fill amb aquest nom.

Finalment, probablement podem reconèixer en dona Fdtima d'Almeria, a qui el setembre de 1444 la reina Maria trameté una carta de resposta a una de seva, la mare de Yusuf i germana de Muhammad IX, que, com hem dit, segons Ibn Asim era Fatima al-Hurra ${ }^{26}$.

Sempre d'acord amb la versió d'Ibn Asim, la documentació catalana també ens permet precisar la data de proclamació de Yusuf $V$, que fins ara es considerava anterior al 15 d'agost de $1445^{27}$, perd que ha de ser anterior al 20 de febrer d'aquest mateix any. El 20 de febrer de 1445, la reina Maria s'adreçà a don Iuce com a rey de Granada, tot contestant-li una carta previa seva en que li devia haver comunicat, com permet interpretar el seu contingut, que havia estat entronitzat poc abans. La carta deixa constància, a més, que Yusuf havia sol licitat a la muller d'Alfons el Magnànim una galera, potser, encara que no necessàriament, amb finalitats militars:

en lo de la galea que nos demandays, al present no la havemos, pero cascún día van e vienen; si, quando n'i haurá alguna, haurá aquí por vós

${ }^{23}$ ARV, Batllia, Lletres i Privilegis, 1148, fols. 465 v. 466 r.

${ }^{24}$ ARV, Mestre Racional, 56, fol. 282 r. Àpoca del 28 d'octubre de 1440. Citat per J. HINOJOSA, Las relaciones, p. 99, text corresponent a la nota 2.

${ }^{25}$ ACA, C., reg. 3190 , fol. 28 v. 1443, descmbre, 23. València.

${ }^{26}$ ACA, C., reg. 3190 , fol. 140 r. 1444, setembre, 22. València. Vegeu M. CharoultT, Edición y estudio, p. 156.

${ }^{27} \mathrm{Cf}$. M. GASPAR, Translado sinple de nonbrainiento. 
qui contracte com el patró de la galea, de buena voluntat vos complazeremos e daremos orden que la hayas ${ }^{28}$.

Dos mesos després, el 24 d'abril, en adreçar-li una recomanació en favor del mercader Antoni Sant Mateu, la reina Maria es dirigia de nou a don Iuceff ben Azmet com a rei de Granada, però aquest cop especificant, també, que ho era de Màlaga, d'Almeria i de Guadix ${ }^{29}$.

\title{
4. La inconsistència del suposat Muhammad X ibn Utman el Coix
}

Cal recordar, i ho podem fer citant paraules del propi Seco de Lucena, que

\begin{abstract}
Muhammad X El Cojo es el único monarca nasri de quien no poseemos más noticias ciertas que las que nos dan los cronistas castellanos, quienes nos cuentan que era sobrino de El Zurdo, hijo de un tal Utman (Ozmin, dicen los referidos cronistas) y que en Granada lo apellidaban El Cojo. No poseemos ninguna fuente árabe que nos suministre información acerca de este monarca y en consecuencia sólo es lícito concluir que ignoramos su verdadera personalidad y que únicamente sabemos, con relativa certeza, que se llamó Muhammad, que ocupó el $X$ lugar entre los que tuvieron tal nombre y que aparece citado en las crónicas castellanas por el mote de $E l$ Cojo. Fue proclamado en una fecha que tampoco conocemos con exactitud, pero que podemos situar en los comienzos del año $1445^{30}$.
\end{abstract}

En l'actualitat, podem seguir subscrivint plenament aquestes afirmacions de Seco de Lucena.

${ }^{28}$ ACA, C., reg. 3195 , fol. 7 r. 1445, febrer, 20. València.

${ }^{29}$ ACA, C., reg. 3193 , fol. 45 r. 1445, abril, 24. València. En aquesta ocasió, la reina Maria intitula Yusuf ibn Ahmad don luceff ben Azınet ben Alahulafe Arraxidin (<al-Khulafa ar-Raixidun?). La cancelleria catalano-aragonesa devia extreure aquesta denominació de la carta que li havia enviat el propi Yusuf; hem d'entendre que aquest, com a descendent de Yusuf II, es considerava representant de la branca legítima de la dinastia contraposant-se a l'usurpador Muhammad IX, descendent de Nasr? He d'agrair, aqui, les observacions i suggeriments interpretatius del Dr. Manuel Sánchez Martínez i de Carles Vela i Aulesa.

${ }^{30}$ Luis SECO DE LUCENA PAREDES, Muhammad IX sultán de Graneda, Granada, 1978, pp. 205-206 (es tracta d'una obra pòstuma que rebé el premi Luis Vives 1957 del CSIC; segurament és una versió redactada a finals dels anys cinquanta que després no fou posada al dia; $c f$. J.-E. LOPEZ DE COCA, Revisión, pp. 61-63). 
Entre les fonts cristianes que s'han anat donant a coneixer, no ha aparegut, fins ara, cap document que faci referencia directa $\mathrm{i}$ explícita a un Muhammad X el Coix, suposat fill d'Utman. La documentació d'arxiu del període cronologic en que se'l situa (1444-1447/1) parla només de Yusuf $^{32}$, d'Ismail ${ }^{33} 0$, genericament, del rei o dels moros de Granada ${ }^{34}$.

Tampoc no ha aparegut cap notícia procedent de fonts àrabs.

I, per acabar, la Yunna no en parla: Ibn Asim no esmenta la rebellio de Muhammad X que, suposadament, consta a les fonts castellanes ${ }^{35}$.

Per tant, cal recórrer directament a les croniques castellanes per localitzar les referencies que la historiografia ha atribuiit a Muhammad X el Coix.

I, en fer-ho, podem constatar tot seguit que, sorprenentment $i$ en contra del que s'ha escrit tantes vegades, ni la Crónica de Don Juan II de Pérez de Guzmán, ni la d'Álvar García de Santamaría, ni la de l'Halconero, redactades a la segona meitat segle XV, no parlen mai de Muhammad el Coix, sinó que sempre esmenten, només, un infant Coix, nebot de Muhammad IX, sense donar-li cap nom ${ }^{36}$.

La Crónica de Pérez de Guzmán, en registrar el suposat alçament de Muhammad X el Coix del 1445, diu, únicament, que

estando el Rey en Villanueva, fué certificado como el Infante Coxo, sobrino de Don Mahomad, Rey de Granada, que llamaban el Izquierdo, hijo de su hernnano, se movió en Almería con trato que había con los

${ }^{31} C$ C. J.-E. LOPEZ DE COCA, Revisión, pp. 64-70.

${ }^{32}$ Vegeu M. GASPAR, Translado sinple; J. HINOJOSA, Las relaciones, pp. 127 i 140-142, documents 18 i 19, i la nova documentació que hem assenyalat a l'apartat anterior.

${ }^{33}$ Vegeu J.-E. LOPEZ DE COCA, Revisión, 67.

${ }^{34}$ Vegeu, per exemple, Juan TORRES FONTES, Don Pedro Fajardo, Adelantado Mayor del Reino de Murcia, Madrid, 1953.

${ }^{35}$ M. Charouiti, Nuevos datos, p. 474, nota 26.

${ }^{36}$ Vegeu Crónica de Don Juan II atribuïda a Pérez de Guzmán, edició Biblioteca de Autores Españoles, vol. LXVIII, Madrid, 1953, pp. 638 i 650 (la p. 654 només esmenta los Moros), en fer referència als esdeveniments de 1445 i 1446; Crónica del Rey don Juan II de Castilla d'Álvar GARCIA DE SANTAMARIA, ed. Colección de documentos inéditos para la historia de España, Madrid, 1891, vol. C, p. 300 en fer referència a la Batalla de la Higueruela (1431) i pp. 364-368 en narrar la recuperació de la capital granadina, des de Malaga, per part de Muhammad IX, després de l'alçament de Yusuf ibn al-Mawl; i Crónica del Halconero de Juan II, ed. Juan de Mata CARRIAZO, Madrid, 1946, p. 122, en fer refertncia a la fugida de Granada de Muhammad IX pel mateix motiu. 
moros de la cibdad de Granada; é vino á la cibdad de Granada, y entró en ella é apodenóse del Alhambra, é prendió al Rey su tio e llamóse Rey,

i que, posteriorment, l'infant Ismail, procedent de Castella i amb el suport castellà,

lanzó fuera al Infante $\operatorname{Coxo}^{37}$.

Quan dóna notícia de la presa de Benamaurel i de Benzalema, el 1446, la Crónica diu només, de nou, que

estando el Rey Don Juan de partida de la villa de Berlanga para ir sobre la villa é castillo de Atienza, le vinieron cartas de la frontera de los Moros, haciéndole saber como el Infante Coxo habia tomado las villas é castillos de Benamaurel é Benzalema ${ }^{38}$.

En canvi, en fer referència a la presa d'Arenas i dels Vélez, únicament esmenta, genericament, los Moros, sense parlar, ni tan sols, del rei de Granada:

Los Moros (...) tomaron en este año [1446], allende las dichas villas de Benamaurel é Benzalema, la villa é fortaleza de Arenas, é la villa é fortaleza de Huesca, é las villas é fortalezas de Velez el Blanco, é Velez el Rubio ${ }^{39}$.

Semblantment, quan Garcla de Santamaria relata la Batalla de la Higueruela, del 1431, on es considera que Muhammad X el Coix capitanejà les forces de Muhammad IX, no diu res més que

el Rey don Mahomad Abenazar el ezquierdo, (...) con temor de los suyos, que despues no le acogerian en la ciudad, ó en el Alhambra, non osó salir á la batalla, é envió á ella un Infante, su sobrino, que decian el Cojo ${ }^{40}$.

${ }^{37}$ Crónica de Don Juan II, p. 638.

38ibidem, p. 650.

${ }^{39}$ ibidem, p. 654.

${ }^{*}$ A. Garcla de Santamaria, Crónica, vol. C, p. 300. 
La Crónica del Halconero, en descriure la fugida de Muhammad IX, el 1432, després de l'alçament de Yusuf ibn al-Mawl, amb dos fills del ja difunt Muhammad VIII el Petit, afegeix només que

llebó consygo a su hermana del ynfante Coxo su sobrino"1.

El 1432, en narrar el procés de recuperació del tron per part de l'Esquerrà, refugiat a Màlaga, García de Santamaría destaca diverses vegades la presència d'aquest seu nebot, l'infant Coix, però sense donar-li mai un nom:

En el mes de Hebrero deste año fué acogido el Infante Coxo, sobrino del Rey ezquierdo, en la ciudad [de Granada] con fasta quinientos moros de caballo é otros tantos de pie que consigo trajo de Málaga, por algunas puertas que le dieron los que eran de su parte ${ }^{42}$.

Luego que este Infante Coxo entró en Granada, el Rey Abenalmao envió (...) á les rogar [als castellans] muy afincadamente que le fuesen á socorrer, que estaba en muy gran peligro ${ }^{43}$.

E en el lugar donde fuera la batalla de que hicimos mencion que el Rey venciera el año pasado [Batalla de la Higueruela, 1431], salió el Infante Coxo con fasta quinientos de caballo é otros tantos peones que consigo dixiemos que había traído de Málaga, é con todos los más de Granada, de caballo é de pie, de la parte del Rey ezquierdo, é pelearon los moros de las dos batallas delanteras dol Adelantado; é como quier que ellos asaz peleaban é recio pero los que venían con el Infante Coxo eran tantos $(\ldots)^{4}$.

Despucs, en sus batallas bien ordenadas, [el Adelantado] volvióse á Pinos, é en llegando á la sierra, sopo que los peones moros del Infante Coxo, é con ellos pieza de los de caballo, venían á tomar la sierra é la Puente de Pinos ${ }^{45}$.

\footnotetext{
${ }^{41}$ Crónica del Halconero, p. 122.

${ }^{12}$ A. Garcia de Santamarfa, Crónica, vol. C, p. 364.

${ }^{43}$ ibidem.

Mibidem, p. 365.

4sibidem, 366.
} 
I, en darrer terme, la mateixa Crónica de García de Santamaría explica que, un cop l'Esquerrà ja era de nou a l'Alhambra, en saber que el Mestre de Calatrava havia entrat a talar Guadix i la seva comarca i que havia assentat el seu reial a una llegua de la ciutat, el juny de 1432

vino á esta ciudad [Guadix] é á su comarca el Infante cojo de Granada, con los más é mejores caballeros de la casa de Granada é de Baza, con fasta dos mil de caballo, é mucha gente de pie, por embargar la tala ${ }^{46}$.

D'on sorgeix, doncs, aquest Muhammad el Coix, fill d'un germà de Muhammad IX anomenat Utman, que no apareix a les croniques del segle XV?

L'assimilació de l'infant Coix amb un nebot de Muhammad IX anomenat Muhammad i fill d'un Hozmén o Hozmin apareix tant a la Historia de la Casa Real de Granada, anonim de mitjan segle XVI editat per Carriazo ${ }^{47}$, com al Compendio de Garibay, publicat a Amberes el $1571^{48}$ perd ja enllestit el $1566^{49}$, dues obres indubtablement emparentades ${ }^{50}$.

Si bé la Historia anonima de la Casa Real de Granada no esmenta les seves fonts d'informacio, Garibay cita, en canvi, Marineo Sículo ${ }^{\mathbf{1 1}}$.

Esperant trobar-hi alguna referència a aquest Muhammad, hem intentat resseguir les obres de Marineo Sículo, perd, entre les que hem pogut tenir a l'abast (De primis Aragonie regibus ${ }^{22}$ i Opus de rebus Hispaniae memorabilibus ${ }^{33}$ ), no hi hem sabut localitzar cap mencio d'aquest infant de Granada, potser continguda a De Hispaniae laudibus libri VII,

\footnotetext{
${ }^{46}$ ibideln, p. 368.

"Juan de MAta CarRazo, La "Historia de la Casa Real de Granada", anónimo castellano de mediados del siglo XVI, "MEAH" VI (1957), 7-56; reeditat dins En la frontera de Granada. Homenaje al profesor Carriazo, Sevilla, 1971, vol. 1, pp. 145-192, pp. 178-179 (citem segons la reedició).

${ }^{48}$ Esteban de GaRIBay ZamalloA, Compendio historial de las chrónicas y universal historia de lodos los reynos d'Espanta, Amberes, 1571.

${ }^{49} \mathrm{Cf}$. J. de M. Carriazo, La "Historia, p. 147.

soibidem, pp. 145-151.

${ }^{\text {st }}$ Cf. ibidem, 149.

${ }^{32}$ Lucio MARINEO SICULO, De primis Aragonie regibus, Saragossa, 1509.

${ }^{33}$ Lucio MARINeO SICULO, Opus de rebus Hispaniae Memorabilibus, Alcalà, 1533.
} 
que fins ara ens ha estat impossible de consultar ${ }^{54}$. En tot cas, per tant, haurem de deixar per futures ocasions l'intent de localitzar l'origen de l'assimilació d'aquest Hozmén amb un Muhammad.

En canvi, per altra banda, podem constatar que, si bé la Descripción general de Áffrica de del Mármol fa esment d'un nebot de l'Esquerrà, fill d'Odman (ja no d'Hozmén ni d'Hozmin), su hermano, que ya era muerto, llamado Muley Mahamete ibni Odman, en aquesta obra no rep mai l'apel-latiu de Coix ${ }^{5 s}$.

Sembla, per tant, molt possible, que l'associació de l'infant Coix amb un Muhammad, que no apareix a les croniques del XV, pot ser posterior, probablement de l'inici o de mitjan segle XVI; altrament, hauria de procedir d'una font que, en principi, ens és desconeguda. Tot indica que és des d'aleshores en endavant que la historiografia es fa resso de l'existència d'un Muhammad el Coix.

Encara que alguns historiadors no hagin deixat de manifestar la seva sorpresa davant d'un nom com el d'Utman, que podria derivar, probablement i progressiva, de l'Hozmén o Hozmín present a Garibay i a la cronica anonima ${ }^{\text {s6, }}$

\begin{abstract}
Otsman, nombre tan extraño en la casa real nazarí que sorprende al propio Lafuente Alcántara y le obliga a decir que es el único rey que no ha podido justificar con documentos arábigos y que solamente por probable conjetura le coloca en su cuadro genealógico ${ }^{57}$,
\end{abstract}

\footnotetext{
${ }^{34}$ Lucio Marineo SICULO, De Hispaniae laudibus libri VII, d'any insegur però, segons sembla, anterior a la mort d'Isabel I (1504). Cf. B. SÁNCHEZ ALONSO, Fuentes de la historia española e hispanoamericana, Madrid, 1952, 3a. edició corregida i actualitzada, vol. I, p. 250.

${ }^{35}$ Luis del MÁRMOL, Descripción general de Affrica con todos los successos de guerras, Granada, 1573, vol. 1, fols. $223 \mathrm{r}$ - v.

${ }^{56}$ Encara que sigui poc probable, no ens podem estar, aquí, d'apuntar una hipòtesi que, si més no, es mostra suggeridora. Com veurem, per ini el Coix no pot ser altre que Yusuf ibn Ahmad. A la documentació cristiana, almenys a la catalano-aragonesa, com a malformació d'Ahmad hi podem trobar Azonet o, fins i tot, Hazmet. Fins a quin punt, doncs, Hozmén no podria ser fruit d'una mala lectura d'Hazmet? Tanmateix, si bé la confusió d'una $a$ i d'una $o$ fóra facil d'explicar, la d'una $t$ i d'una $n$ seria més difícil. L'atribució que se li fa del nom de Muhammad ja seria una altra cosa; peró, tot $i$ això, els errors de les cróniques $i$ de la historiografia de l'época en el parentiu i, adhuc, en la identitat dels personatges, és un fet ben notori.

${ }^{57} \mathrm{Cf}$. Mariano GASPAR REMIRO, Investigaciones sobre los Reyes de Granada. ¿Quién fue el sultán Yuzef Aben-Almaul o Aben-Almao de nuestras crónicas?, dins Con motivo del Romancero..., p. 143, reedició de "Revista de Libros" II (1914), p. 6.
} 
uns altres autors han arribat esmentar el Coix pel seu nom complet -Abu Abd Allah Muhammad ibn Utman ibn Nasr ibn Muhammad V-i li han atribuït l'apel-latiu en àrab, al-Afnah ${ }^{58}$.

Tanmateix, i mentre no apareguin testimonis en sentit contrari, el que sí podem afirmar és que l'existència d'un Muhammad el Coix, fill d'un Utman germà de Muhammad $\mathrm{IX}^{59}$, no té, pràcticament, cap consistència; perque, en cap cas i sense excepcio, els fragments de les croniques del XV que habitualment $s$ 'han citat $o$ adduït per fer-hi referència no l'esmenten mai com a tal, perque sempre parlen, només, d'un infant Coix ${ }^{60}$; finalment, podem afegir que l'única evidencia que en tenim fins ara procedeix de dues obres dels inicis de la segona meitat del segle XVI, pero que l'anomenen Muhammad ibn Hozmén o Hozmin, no pas ibn Utman.

\section{Fou Yusuf IBN AHMAD L'INFANT COIX DE LES CRÒNIQUES? NIHIL OBSTAT.}

Un cop determinat, sense cap mena de dubte, que Yusuf ibn Ahmad i Aben Ismael no foren una mateixa persona ${ }^{61}$ i, per altra banda, que no hi ha cap evidencia ferma que ens permeti considerar que l'infant Coix de les croniques s'anomenava Muhammad, es fa necessària una relectura d'aquestes i una revisió de totes les dades disponibles dels tres personatges.

En primer lloc, seguint els esdeveniments que narra la Yunna, podem observar que les notícies documentals i cronístiques que fan referència explícita a Ismail concorden perfectament amb les d'Ibn Asim: Ismail,

\footnotetext{
${ }^{58}$ Vegeu L. SECO DE LUCENA, Más rectificaciones, pp. 281 i 284, i també Rachel ARIE, L'Espagne musulmane au temps des nasrides (1232-1492), Paris, 1990, p. 138 i nota 5.

${ }^{39}$ No cal dir que la suposada existència d'aquest Utman ve determinada per la tambe suposada existencia d'un Muhaminad ibn Utman, i que, si Muhammad ibn Utman el Coix desapareix, també ho fa, al seu darrera, aquest suposat germà de Muhammad IX.

${ }^{60}$ Entre els exponents d'aquestes múltiples citacions directes però inexactes dels fragments de les cròniques castellanes transcrits més amunt, vegeu, per exemple, L. SECO DE LUCENA, Nuevas recrificaciones, pp. 400 i 401 ; L. SECO DE LUCENA, Más rectificaciones, pp. 280, 281 i 282; i H. LIVERMORE, El segundo rey Chico, p. 332, tot i que són presents ja en la historiografia anterior.

${ }^{61}$ Assimilació que, com la de l'infant Coix amb Muhammad X, tambe s'havia establert amb pocs fonaments -perque tampoc no hi havia cap noticia que identifiques mútuament tots dos personatges (vegeu L. SECO DE LUCENA, Una rectificación)- i que havia hagut de donar pas a autèntics malabarismes per salvar les contradiccions $\mathrm{i}$ per conciliar totes les notícies disponibles (vegeu J.-E. LOPEZ DE COCA, Revisión, pp. 64-67).
} 
un infant que residia a la cort de Joan II, apareix a la documentacio, recolzat pel rei de Castella i cridat pels detractors de l'infant Coix, entre 1446 i $1447^{62}$, i hi reapareix el $1450^{63}$.

Posteriorment, una analisi comparada de les dades que Ibn Asim dóna de Yusuf $\mathrm{i}$ de les notícies que les croniques castellanes transmeten de l'infant Coix, alliberat ja del nom de Muhammad, permet descobrir, sense obstacles, que podrien ser una mateixa persona. Perque, mentre que els paral-lelismes i les coincidencies entre totes dues trajectories són inqüestionables, no hi ha cap discrepancia que impedeixi identificar-los. Ben al contrari: les notícies de Yusuf ibn Ahmad i les de l'infant Coix són concordants i facilment conciliables; les dades que forneix la Yunna i les de les cròniques castellanes poden ser, fins i tot, mútuament complementàries; i, adhuc, podem concloure que les cròniques castellanes son, en aquest cas, del tot verídiques i que, pràcticament, no incorren en cap error.

Vegem, ara, com encaixen totes les peces d'aquest trenca-closques, i quina és la reordenació de les dades i la relectura del perfode que s'imposa si donem per vàlida la hipotesi que Yusuf ibn Ahmad és l'infant Coix de les croniques castellanes.

\section{Yusuf V ibn Ahmad el CoIX, ReI de Granada: REORDENACIÓ DE LES DADES I RELECTURA DEL PERÍODE}

Si Yusuf V de Granada era, com diu Ibn Asim, nebot de Muhammad IX l'Esquerrà, perquè era fill de la seva germana Fatima al-Hurra ${ }^{64}$, Fatima al-Hurra podria ser la dona Fatima d'Almeria a qui, el setembre de 1444, la reina Maria, muller d'Alfons el Magnànim, adreçava una carta en resposta a una de seva ${ }^{65}$.

Segurament, com afirma la Crónica del Halconero, quan Muhammad IX abandonà l'Alhambra, el 1432, després de l'alçament de Yusuf ibn al-Mawl, enduent-se'n dos fills petits del ja difunt Muhammad VIII el Petit,

\footnotetext{
${ }^{62}$ Vegeu J.-E. LOPEZ DE COCA, Revisión, pp. 66-67.

${ }^{63}$ ibidem, pp.72-75.

at M. Charouitt, Edición y estudio, p. 156.

${ }^{65}$ Vegeu la nota i el text corresponent a la nota 26.
} 
la seva germana Fàtima l'acompanyà (llebo consygo a su hermana del ynfante Coxo su sobrino ${ }^{66}$ ).

Pero aixo no és cap impediment perquè el pare de Yusuf $\mathrm{V}$ fos l'infant Ahmad ibn Yusuf II, com mostra el trasllat del document publicat per Gaspar Remiro ${ }^{67}$ i com corrobora la documentació catalano-aragonesa adreçada a l'infant don Iuceff, fill del infant Azmet o al rei don Iuceff ben Azmet ${ }^{68}$.

D'aquesta manera, Yusuf ibn Ahmad, besnét de Yusuf II per part de pare, seria fruit de la unio de la branca legítima destronada per Muhammad IX i de la il·legitima, descendent de Nasr, entronitzada amb l'usurpació de l'Esquerrà.

Aleshores, les croniques castellanes no estarien equivocades quan afirmen que l'infant Coix era nebot directe de Muhammad IX ${ }^{69}$; l'únic error apareixeria a la Crónica de Pérez de Guzmán quan diu, en una sola ocasio, que el Coix era fill d'un germà, i no pas d'una germana, de l'Esquerrà $^{70}$.

Com mostra Ibn Asim, Yusuf el Coix no es refugià mai a la cort castellana. Féu costat al seu oncle a la Batalla de la Higueruela, capitanejant les forces musulmanes ${ }^{71}$, i posteriorment, el 1432, des de Màlaga, on probablement s'havia refugiat amb el seu oncle i la seva mare, l'ajuda, com relata a bastament García de Santamaría, a recuperar el poder que li havia usurpat Yusuf ibn al-Mawl amb suport castella ${ }^{72}$. Un cop l'Esquerra ja fou de nou a l'Alhambra, possiblement el juny del 1432, comanda les forces que anaren a socórrer Guadix, amenaçada pel Mestre de Calatra$\mathrm{va}^{73}$.

Incentivat, segons la Yunna, pels enemics del seu oncle, es retirà a Huétor Vega a causa dels problemes que sorgiren en llurs relacions

\footnotetext{
${ }^{66}$ Crónica del Halconero, p. 122.

${ }^{67} \mathrm{M}$. GASPAR, Translado sinple

${ }^{68}$ Vegeu les notes $i$ el text corresponent a les notes 22 i 27-29.

${ }^{69}$ Vegeu les citacions corresponents a les notes 40 i 42.

${ }^{70}$ Vegeu la citació corresponent a la nota 37.

"Vegeu la citació corresponent a la nota 40.

${ }^{2}$ Vegeu les citacions corresponents a les notes 42 a 45 .

${ }^{73}$ Vegeu la citació corresponent a la nota 46.
} 
mútues $^{74}$. Pero, gràcies a la intervenció de la seva mare Fàtima, fou nomenat alcaid d'Almeria, cosa que produr uns anys de calma ${ }^{75}$. Les notícies que en tenim com a infant d'Almeria, directes entre finals de 1438 i 1441 $i$ indirectes -a través de la seva mare i d'un seu fill anomenat Muhammadfins al setembre de 1444, deuen correspondre a aquest perfode de tranquillitat $^{76}$.

Essent una generació posterior a Muhammad IX i fill d'un infant que, el 1410 , ja havia socorregut Antequera ${ }^{7}$, el 1431 ja podia perfectament tenir edat suficient com per protagonitzar els fets que des d'aleshores se li atribueixen $i$, a la fi de 1443 , per tenir un fill adult ${ }^{78}$.

A principis de 1445, abans del 20 de febrer, Yusuf el Coix s'hauria alçat definitivament contra el seu oncle $i$, almenys des del mes d'abril, ja s'intitulava rei de Granada, de Màlaga, d'Almeria i de Guadix ${ }^{79}$. El 15 d'agost d'aquell mateix any, des de l'Alhambra, nomenava un alcaid a Almeria ${ }^{80}$.

Si Yusuf $\mathrm{V}$ fou l'infant Coix, les cròniques no s'equivocarien quan, coincidint amb la versió d'Ibn Asim, diuen que l'infant Coix, que residia a Almeria, s'alçà contra el seu oncle d'acord amb alguns cavallers granadins, s'apoderà de la capital i de l'Alhambra i l'empresona ${ }^{81}$, primer a Salobreña i després a Almeria

Un cop Yusuf $\mathrm{V}$ fou al tron, aparegué, per primer cop, també a principis de 1445, Abu 1-Walid Ismail, que, tanmateix, en aquella ocasio, sembla que no va arribar a Granada; després que Yusuf sofoqués les revoltes de la capital i restablís l'ordre, Ismail hagué de tornar a Castella ${ }^{83}$.

${ }^{74}$ M. ChAROUIT, Edición y estudio, p. 157.

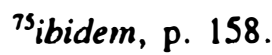

${ }^{76}$ Vegeu les notes $\mathrm{i}$ el text corresponent a les notes 22 a 26.

${ }^{17} \mathrm{Cf}$. M. GASPAR, Investigaciones sobre los Reyes, p. 143. Tanmateix, per recolzar que Ahmad i Ali (el pare de Sad), germans de Yusuf III, socorregueren Antequera, se cita habitualment un fragment de la Crónica de Pérez de Guzmán que diu, únicament, que el rey de Granada, coino supo que el Infante [Ferran de Castella] estaba sobie Antequera, inandó a dos Infantes, sus hermanos (cf. Crónica de Don Juan II, p. 318), sense anomenar-los.

${ }^{78}$ Vegeu el text corresponent a la nota 25.

${ }^{79}$ Vegeu el text corresponent a les notes 28 i 29.

${ }^{80}$ M. GASPAR, Translado sinple.

${ }^{81}$ Vegeu la primera citació corresponent a la nota 37.

${ }^{82} \mathrm{M}$. Charouiti, Edición y estudio, p. 161.

${ }^{83}$ ibidem, pp. 161-162. 
Un cop Ismail fou de nou a Castella, els partidaris de Muhammad IX haurien proposat al monarca que el cridés per acabar amb Yusuf; ell s'hi hauria oposat. Pero, a mitjan febrer de 1446, Ibrahim ibn Abd al-Barr li hauria ofert el seu ajut. Aleshores Ismail, recolzat per Abd al-Barr i els abencerratges, que l'haurien recolzat esperant que, un cop aconseguit el poder, restituiria l'Esquerrà al tron, va aconseguir allo que es proposava. Tres dies després de la seva arribada, Yusuf va abandonar l'Alhambra i es va refugiar a Almeria amb els seus addictes. Ismail hauria regnat des del 12 de febrer de 1446 fins al 13 d'agost de 1447. Aquell mateix dia hauria arribat la notícia de l'assassinat de Yusuf ibn Ahmad per part del seu ministre Ali ibn Allaq. Un mes després, Muhammad IX hauria recuperat el tron. Aleshores, les noticies d'Ismail desapareixen fins a 1450 , quan retorna per tercer cop $^{84}$.

Aquesta versió concorda també, perfectament, amb la de la Crónica de Pérez de Guzmán, segons el qual, després de la presa de poder de Yusuf, el 1445 ,

el Alguacil mayor llamado Andilbar, á quien desto mucho pesó, é algunos otros cavalleros sus parientes, se vinieron á Montefrio, que es cerca de Alcalá la Real, y embiaron luego dos mensageros á Castilla al Infante Izmael que era con el Rey; con los quales le embiaron decir que se fuese para ellos é que lo tomarian por Rey; é como aquellos mensageros le llegaron, el Infante lzmacl, que era vasallo del Rey, le demandó licencia para se ir para Granada, certificándole que si oviese el Reyno le serviria siempre con él é seria su vasallo. El Rey le dió licencia, é le mandó dar gente é dineros con que se fuese, é fué rescebido por Rey en Granada, é lanzó fuera al Infante Coxo, segun la historia adelante lo contarás ${ }^{85}$.

Les cartes d'Ismail i de l'alcaid de Gibraltar, Audalla Abenyasis, sol-licitant l'alliberament de dos moros gibraltarencs que havien estat conduïts a Sevilla, llegides al capitol celebrat a Jérez el 27 de febrer de 1446, queden correctament inserides, per tant, en el curt regnat d'Ismail ${ }^{86}$.

La documentació d'arxiu castellana també ens permet confirmar el suport castellà a Ismail que esmenta Pérez de Guzmán; és probable que Ibn Asim no en parli per protegir Muhammad IX, tot evitant que es pogués

\footnotetext{
3ibidem, pp. 163-164.

${ }^{85}$ Crónica de Don Juan II, p. 638.

${ }^{86} \mathrm{~J} .-\mathrm{E}$. LÓPEZ DE COCA, Revisión, p. 67.
} 
considerar que, per bé que indirectament, a través d'Ismail, havia acceptat el recolzament cristia. El 15 de febrer i el 3 d'abril de 1447, el rei de Castella escrigué al consell de Sevilla $i$ al de Jérez, respectivament, per informar-los que Ismail, rei de Granada, vassall seu, li havia demanat ajut en contra de l'infant Coix de Granada, que era dado e se dava cada dia favor e ayuda por algunos christianos, mis rebeldes de los dichos mis regnos $^{87}$.

Com diu López de Coca, la col·laboració del Coix amb els cristians fou real, i cal relacionar-la amb els esdeveniments murcians ${ }^{88}$.

I Luis del Mármol podria tenir, cojunturalment, raб, quan afirma que

Aben Odman rey de Granada [ $=$ Yusuf $V$ el Coix] hizo paz y aliança con

Don Juan rey de Navarra contra don Juan rey de Castilla, que favorescía a Ismael ${ }^{89}$,

ja que, a més, ens consta que, en arribar al poder, Yusuf havia demanat ajut, o almenys una galera, a la reina Maria ${ }^{90}$.

D'acord amb la Yunna, les croniques tampoc no s'equivocarien en atribuir a l'infant Coix, és a dir, a Yusuf $\mathrm{V}$ i no pas, com es creia, al suposat Muhammad X, la recuperació de Benamaurel, Benzalema, Arenas, Huéscar i un dels Vélez -no pas tots dos ${ }^{91}$.

Ara també podem reafirmar amb certesa, gràcies a Ibn Asim, que Muhammad IX recuperà el tron abans de l'octubre de $1447^{92} \mathrm{i}$, en concret, entre els mesos d'agost i setembre d'aquell any ${ }^{93}$.

Seria aleshores, el 1447, després que l'Esquerrà hagués recuperat el tron, quan, segons la Yunna, el veritable Muhammad X, fill de Muhammad VIII el Petit, el Chiquito de les fonts castellanes, apareixeria a la vida

\footnotetext{
${ }^{87}$ ibidem, p. 67 i nota 21.

${ }^{8}$ ibidem, p. 68. Vegeu també J. TORRES, Don Pedro Fajardo, pp. 21-42; idem, La intromisión.

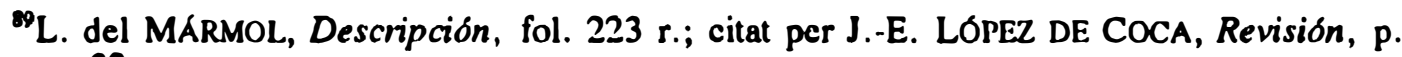
71 , nota 32.

${ }^{90}$ Vegeu el text corresponent a la nota 28.

${ }^{91} \mathrm{M}$. Charourn, Edición y estudio, pp. 183-185, i vegeu les citacions corresponents a les notes 38 i 39.

22J.-E. LÓPEZ DE COCA, Revisión, p. 68.

${ }^{93}$ M. Charouith, Edición y estudio, p. 164.
} 
política granadina, com a braç dret de Muhammad IX. Com hem dit, la gran entesa entre tots dos hauria fet que es casés amb una filla de l'Esquerrà, Umm al-Fath ${ }^{94}$. Aixf, de nou, com en el cas de Yusuf V, Muhammad IX hauria aconseguit, amb aquest casament, unir les dues branques de la famflia, tot intentant assegurar l'estabilitat interna. I, d'aquesta manera, hauria quedat amb les mans lliures per prosseguir la politica exterior agressiva que palesen les fonts cristianes al llarg dels mesos següents ${ }^{95}$.

Tanmateix, Muhammad IX no hauria tardat gaire en tornar a perdre el poder. Entre març i abril de 1450, Ismail, procedent de Castella i amb nou recolzament cristia ${ }^{\circ}$, hauria reaparegut per tercera $\mathrm{i}$ última vegada. Instal-lat primer a Comares, el 3 d'abril hauria ocupat l'Alcazaba de Màlaga, despertant el terror a la capital granadina. Segons Ibn Asim, els alfaquís haurien aconsellat al poble que fos fídel a Muhammad IX, més digne del seu suport que no pas Ismail, que havia pactat amb els infidels. I, d'aquesta manera, l'Esquerra hauria pogut preparar, amb llur aprovació, les operacions contra l'usurpador' ${ }^{97}$.

Després de la recuperació de Vélez-Màlaga i de la conquesta, el 26 de juny, de la ciutat de Màlaga ${ }^{98}$, els partidaris d'Ismail, encastellats a l'Alcazaba, haurien acabat rendint-se ${ }^{99}$. Dos dies més tard, l'Esquerrà hauria rebut l'acatament de la ciutat i de la part occidental del regne $i$, el 30 de juny, Ismail hauria mort ${ }^{100}$.

El primer dia de juliol de 1450, l'endemà de la que, segons Ibn Asim, és la data de la mort d'Ismail, la notícia encara no havia arribat a Sevilla. Pero se sabia que Ismail, que havia demanat ajut a Castella, estava

94ibidem, p. 170.

95J.-E. LOPEZ DE COCA, Revisión, pp. 70-72.

${ }^{96}$ El 31 de maig de 1450 Per Afán de Ribera infonnà el consell sevillà de les gestions realitzades amb Ismail per encàrrec de Joan II, i el 7 d'abril Joan II de Castella ja notificà a les autoritats del regne que li havia atorgat treves a ell $i$ als llocs de Granada que estiguessin sota la seva obediència. CS. ibidem, pp. 72-73, i el document 1 de l'apèndix.

${ }^{97}$ M. Charouiti, Edición y estuclio, p. 164.

${ }^{96}$ Les forees enviades per l'adelantat Per Afán de Ribera a Ismail per socórrer-lo a Malaga es toparen, a mitjan mes de juny, amb les afectes a Muhainmad IX $\mathrm{i}$ a Muhammad el Chiquito, que els tallaren el pas. CY. J.-E. LOPEZ DE COCA, Revisión, pp. 73-74 i document 2 de l'apèndix.

${ }^{99}$ Segons relació de Per Afán de Ribera del 24 de juliol de 1450, la traïció d'un dignatari local, Reduan de las Fijas, va facilitar l'entrada de les tropes de l'Esquerrà i del rei Xic a la ciutat de Màlaga. Cf. J.-E. LOPEZ DL: COCA, Revisión, pp. 74-75. Ibn Asim no ho esmenta.

${ }^{100}$ M. Charouint, Edición y estudio, pp. 164-165. 
perillosament assetjat a l'Alcazaba, i que Gibralfaro també estava en poder seu $^{101}$.

Des d'aleshores i fins a la mort de l'Esquerrà, anterior al 24 de juliol de $1453^{102}$, Muhammad IX hauria regnat amb el seu gendre, Muhammad X, el rei Xic. Llur política agressiva s'hauria estes fins a 1452, quan pactaren treves amb Joan II de Castella, sol-licitades, segons Ibn Asim, pels castellans, a causa de les moltes victories que havia aconseguit el Chiquito ${ }^{103}$.

Tal com succeeix amb Yusuf V, Muhammad ibn Muhammad VIII apareix a la documentació arxivística catalano-aragonesa, entre el mes de maig de 1451 i l'estiu de 1453, com a infant d'Almeria ${ }^{104}$. Tanmateix, de totes les missives adreçades a ell que coneixem, trameses des de Valencia pel batlle general del regne, Berenguer Mercader, n'hi ha, normalment, un duplicat amb el mateix contingut dirigit a Muhammad IX com a rei de Granada.

Després de la mort del seu sogre, Muhammad el Xic hauria esdevingut, com a Muhammad X, no pas com a Muhammad XI, senyor de l'Alhambra ${ }^{105}$; pero no tardaria a tenir problemes amb el cosí-germà del seu pare, Sad, i a perdre la vida a les seves mans ${ }^{106}$.

La possible identificació de Yusuf $\mathrm{V}$ ibn Ahmad amb l'infant Coix de les croniques castellanes, acompanyada de l'evidencia que aquest Yusuf i l'infant Ismail foren dues persones diferents i de les dades que forneix, amb la seva tesi doctoral, malauradament inedita, la Dra. Charouiti, podria deixar closa, al meu entendre, almenys provisionalment $i$-com s'ha hagut

\footnotetext{
${ }^{101} \mathrm{~J} .-\mathrm{E}$. LÓPEZ DE COCA, Revisión, pp. 74-75 i document 2 de l'apèndix. Segons la Yunna, els partidaris d'Isinail demanaren, en negociar llur rendició, que Muhammad IX els cedís les dues alcassabes i Gibralfaro; of. M. CHAROUITT, Edición y estudio, p. 165.

${ }^{102}$ Cf. J.-E. LÓPEZ DE COCA, Revisión, p. 78 i document 3 de l'apèndix. A partir de 1450 la Yunna ja no ens pot ajudar, perque els fets que narra arriben fins a $1450 \mathrm{i}$ el propi lbn Asim morí el 1453; of. M. ChAROUIm, Edición y estudio, pp. 71, 113 i 168.

${ }^{103} \mathrm{M}$. Charoutn, Edición y estudio, p. 170.

${ }^{104}$ Sempre hi figura com a don Mahomat, infant d'Almeria, fill de don Mahomat, rei de Granada. ARV, Batllia, Lletres i Privilegis, 1151, fols. 204 v., 413 v. i 757 v.

${ }^{105 J .-E . ~ L O P E Z ~ D E ~ C O C A, ~ R e v i s i o ́ n, ~ p . ~} 78$.

${ }^{106}$ ibidem, pp. 78-85; H. LIVERMORE, El segundo rey Chico.
} 
de dir tantes vegades- mentre no apareguin testimonis en sentit contrari, la revisió d'aquest període de la historia granadina.

Si Yusuf $V$ és l'infant Coix de Granada que apareix a les cròniques, és evident que, en contra del que es creia fins ara, no passa desapercebut per les fonts castellanes, fet que seria sorprenent si considerem la importància que li atribueix la Yunna d'Ibn Asim i les múltiples notícies arxivistiques que ens n'han pervingut.

Aleshores, malgrat el pes de la seva presencia en la historiografia que pot remuntar-se, almenys, fins a mitjan segle XVI- Muhammad X el Coix ibn Utman ibn Nasr ibn Muhammad $V$ només hauria estat un miratge, generat per l'homonímia dels sultans granadins del segle XV, per les imprecisions en llur successió i pels equfvocs, amb llarga tradició, en l'atribució de llurs apel $\cdot$ latius.

Amb la desaparició de Muhammad X el Coix, Muhammad el Chiquito quedaria convertit en Muhammad X, Boabdil esdevindria Muhammad XI i el seu oncle, Muhammad el Zagal, seria Muhammad XII.

Tanmateix, perd, com ja deia Ladero ${ }^{107}$, la mort de Yusuf $\mathrm{V}$ i d'Ismail abans de 1462 ha deixat oberts nous interrogants, fins ara insospitats, a l'etapa posterior, essencialment pel que fa a la determinació de la personalitat de qui fins ara, i ja en epoca de Sad, s'havia considerat Yusuf $\mathrm{V}$ Aben Ismael i que, des del descobriment de la Yunna, caldria denominar Yusuf VI.

\section{RÉSUMÉ}

L'historiographie avait identifie, traditionnellement, l'infant Cojo de Grenade qui apparait aux chroniques castillanes avec Muhammad X ibn Utman, alors qu'avait assimile Yusuf ibn Ahmad avec l'Aben Ismael des chroniques. La thèse de M. Charouiti, qui édite et étudie une oeuvre du grenadin Ibn Asim, contemporain aux événements, que l'on considérait perdue, démontre que Yusuf et Ismail furent deux personnes différentes. Alors, une revision des données que cet ouvrage nous apporte, de quelques

${ }^{107}$ M.Á. LADERO, Granada, nota de la p. 189. 
nouvelles données inédites des archives catalans et de la présence de l'infant Cojo aux chroniques castillanes, lesquelles ne l'appellent jamais Muhammad, nous permet découvrir que Yusuf V ibn Ahmad pourrait être l'infant Cojo des chroniques, et que la supposition de l'existence de ce Muhammad X el Cojo avait été produite par l'homonymie des sultans grenadins du XVe. siecle, par les imprécisions en leur succession et par les erreurs, avec une longue tradition, dans l'attribution de leurs appellatifs.

\section{SUMMARY}

Historiography has traditionally assumed that the Granada's infante Cojo that appears in the Castilian chronicles was Muhammad X ibn Utman, and it had identifyed Yusuf ibn Ahmad with the also chronistical infante Aben Ismail. M. Charouiti's PHD, that edits and studies a Granada's writer Ibn Asim work that had been considered missing, proves that Yusuf and Ismail were two different people. A revision of the information that this work gives together with new data obtained in catalan archives and of the Cojo's presence in Castilian chronicles, that never designate him as Muhammad, permits to discover that Yusuf V ibn Ahmad could be the chronistical infante Cojo and, in case, that the conjectural existence of Muhammad X el Cojo has been produced by Granada's XVth. century sultans homonymy, by lack of precision in their succession and by the long traditional mistakes commited by attributing them their appellatives. 